\title{
Antistress effects of Kampo medicine "Yokukansan" via regulation of orexin secretion
}

\author{
This article was published in the following Dove Press journal: \\ Neuropsychiatric Disease and Treatment \\ 20 March 2017 \\ Number of times this article has been viewed
}

\author{
Haruto Katahira' \\ Masataka Sunagawa' \\ Daishi Watanabe ${ }^{1,2}$ \\ Yasuaki Kanada ${ }^{1,3}$ \\ Ayami Katayama' \\ Risa Yamauchi' \\ Masashi Takashima ${ }^{4}$ \\ Shintaro Ishikawa' \\ Tadashi Hisamitsu' \\ 'Department of Physiology, \\ ${ }^{2}$ Department of Neurology, School \\ of Medicine, Showa University, \\ ${ }^{3}$ Department of Surgery, Showa \\ University Koto Toyosu Hospital, \\ Tokyo, ${ }^{4}$ Department of Orthopaedic \\ Surgery, Showa University Fujigaoka \\ Hospital, Kanagawa, Japan
}

\begin{abstract}
Objective: Various stressors induce stress responses through the hypothalamic-pituitaryadrenal and the sympathetic-adrenal-medullary axes, which are regulated, in part, by orexin. For example, secretion of orexin in the hypothalamus is increased in rats exposed to the stress of social isolation for 1 week. In this study, the antistress effects of Kampo medicine Yokukansan (YKS) via the regulation of orexin secretion were investigated using a rat model.
\end{abstract}

Methods and results: The administration of $300 \mathrm{mg} / \mathrm{kg}$ per day of YKS to rats for 1 week significantly decreased the plasma orexin levels compared with non-treated rats, whereas the administration of $1,000 \mathrm{mg} / \mathrm{kg}$ of YKS had no effect on orexin levels. Therefore, $300 \mathrm{mg} / \mathrm{kg}$ of YKS was an effective dose for controlling orexin secretion. Subsequently, rats were divided into group-housed control (Con), individually housed stress (Stress), and individually housed YKS $(300 \mathrm{mg} / \mathrm{kg})$-treated stress (Stress + YKS) groups. After 1 week, a resident-intruder aggression test was performed, and the plasma levels of orexin and corticosterone were measured. In the Stress group, aggressive behavior and the levels of corticosterone and orexin significantly increased compared with the Con group; however, these effects were inhibited in the Stress + YKS group. Further, an orexin receptor antagonist (TCS $1102 ; 10 \mathrm{mg} / \mathrm{kg}$ ) was intraperitoneally administered to rats exposed to isolation stress to determine whether orexin was involved in stress responses. Under these conditions, aggressive behavior and the level of corticosterone significantly decreased compared with the Stress group.

Conclusion: These results suggest that orexin is involved in the control of stress response and that YKS exerts an antistress effect via the regulation of orexin secretion.

Keywords: Yokukansan, orexin, antistress effect, social isolation stress, corticosterone, aggressive behavior

\section{Introduction}

Stress causes anxiety, nervousness, and anger as well as physical and behavioral changes. Further, stress affects the autonomic nervous system, endocrine system, and immune system and is involved in the onset and exacerbation of diverse diseases, including psychological disorders such as depression and anxiety. Stressors induce stress responses through the hypothalamic-pituitary-adrenal (HPA) and the sympathetic-adrenal-medullary (SAM) axes. Moreover, orexin is involved in the regulation of these axes. ${ }^{1-3}$

Orexins (also called hypocretins) are neuropeptides, and orexins A and B are derived from the same precursor peptide, prepro-orexin. ${ }^{4}$ Orexinergic neurons are mainly expressed in the lateral and perifornical areas of the posterior hypothalamus, and their axons are widely distributed throughout the central nervous system, except in the cerebellum. ${ }^{4}$ Orexins A and B bind to the orphan G-protein-coupled receptor, orexin-1 receptor $(\mathrm{OX} 1 \mathrm{R})$, which is highly selective for orexin $\mathrm{A}$, and the orexin-2 receptor
Correspondence: Masataka Sunagawa Department of Physiology, School of Medicine, Showa University, I-5-8 Hatanodai, Shinagawa-ku, Tokyo I42-8555, Japan

Tel +8I 337848110

Fax +8I 337845368

Email suna@med.showa-u.ac.jp
Neuropsychiatric Disease and Treatment 2017:13 863-872

863 
(OX2R) is nonselective for orexins A and B. ${ }^{4}$ Orexins are involved in the modulation of multiple physiological processes such as sleep, arousal, feeding, metabolism, ${ }^{3}$ nociception, ${ }^{5,6}$ and in the autonomic regulation of the cardiovascular, ${ }^{7}$ respiratory, ${ }^{8}$ and neuroendocrine systems. ${ }^{9}$

Yokukansan (YKS; Yi-Gan San in Chinese), which was first reported in the Bao-ying jin-jing-lu (written in 1550), is a traditional herbal (Kampo) medicine comprising seven herbs (Table 1). ${ }^{10}$ The herbs are mixed and extracted with purified water at $95.1^{\circ} \mathrm{C}$ for $1 \mathrm{~h}$, and the soluble extract is separated from the insoluble waste and then concentrated by removing water under reduced pressure. The constituents of the YKS extract are identified using threedimensional high-performance liquid chromatographic analysis (Figure 1). YKS is administered to patients with symptoms such as emotional irritability, neurosis, and insomnia as well as to infants who cry at night and experience convulsions. ${ }^{10}$

Studies of animals demonstrate the antidepressant, antiaggressive, and anxiolytic effects of YKS $;^{11-15}$ however, the mechanisms of the psychopharmacologic effects of YKS are incompletely characterized. Further, there are no published studies, to our knowledge, of the effects of YKS on the orexinergic system.

The current study was designed using a rat model of social isolation stress to determine whether the administration of YKS exerts its antistress effect via the regulation of orexin secretion. This animal model is used to study psychological stress induced by a loss of social interaction. Rats live in the wild in groups, and when housed alone, they show stress responses, such as adrenomegaly; increased secretion of adrenocorticotropin (ACTH), corticosterone, and catecholamine; ${ }^{16}$ aggressive behavior; ${ }^{11}$ locomotor hyperactivity $;{ }^{17}$ increase in food intake; ${ }^{18}$ and oxidative DNA damage. ${ }^{19}$ In the current study, to evaluate the antistress effect of YKS, we investigated the aggressive behavior of rats and changes in their food intake, plasma corticosterone levels, and orexin secretion.

Table I Component galenicals of YKS

\begin{tabular}{ll}
\hline Uncariae cum Uncis ramulus & $3.0 \mathrm{~g}$ \\
Cnidii rhizoma & $3.0 \mathrm{~g}$ \\
Bupleuri radix & $2.0 \mathrm{~g}$ \\
Atractylodis Lanceae rhizoma & $4.0 \mathrm{~g}$ \\
Poria & $4.0 \mathrm{~g}$ \\
Angelicae radix & $3.0 \mathrm{~g}$ \\
Glycyrrhizae radix & $1.5 \mathrm{~g}$ \\
\hline
\end{tabular}

Note: Weights show the mixing amounts. Abbreviation: YKS, Yokukansan.

\section{Materials and methods}

\section{Rats}

Male Wistar rats (7-weeks-old, weighing 200-230 g) were purchased from Nippon Bio-Supp. Center (Tokyo, Japan). During the experiment, animals were housed in standard plastic cages (for a group raising in a W $24 \times \mathrm{L} 40 \times \mathrm{H} 20 \mathrm{~cm}$ cage and for a single raising in a W $26 \times \mathrm{L} 26 \times \mathrm{H} 18 \mathrm{~cm}$ cage) and were kept in our animal facility at $25^{\circ} \mathrm{C} \pm 2{ }^{\circ} \mathrm{C}, 55 \% \pm 5 \%$ humidity, with a light/dark cycle of 12 h. Food (CE-2; Clea Japan, Inc., Tokyo, Japan) and water were provided ad libitum. Experiments were performed according to the guidelines of the Committee of Animal Care and Welfare of Showa University. All experimental procedures were approved by the Committee of Animal Care and Welfare of Showa University (certificate number: 02094).

\section{Drugs}

YKS (Lot No 2110054010; Tsumura \& Co., Tokyo, Japan) was dissolved in distilled water and orally administered. TCS 1102, a dual orexin receptor antagonist (DORA; 91614136-1; Tocris Bioscience, Bristol, UK), was dissolved in 50\% dimethyl sulfoxide (049-07213; Wako Pure Chemical Industries, Osaka, Japan) and intraperitoneally administered.

\section{Influence of YKS on orexin secretion}

To determine the influence of YKS on orexin secretion, 18 rats were randomly divided into groups as follows: 1) group-housed control (Con); 2) YKS $300 \mathrm{mg} / \mathrm{kg}$ (YKS300); and 3) YKS 1,000 mg/kg (YKS1000). Rats were housed in groups of three per cage. Doses of YKS, which were chosen according to the results of published studies, ${ }^{11-}$ ${ }^{15,20-26}$ were administered daily for 7 days to the YKS groups, and water was administered orally to the Con group. On day 8 , rats were anesthetized with intraperitoneally administered pentobarbital sodium $(50 \mathrm{mg} / \mathrm{kg}$; Somnopentyl; Kyoritsu Seiyaku, Tokyo, Japan), and blood samples were taken from the inferior vena cava. To avoid influences of daily fluctuation, all blood samplings were performed from 1:00 to 3:00 pm. Plasma orexin A level was measured using an enzyme-linked immunosorbent assay (ELISA) kit (EKE-003-30; Phoenix Pharmaceuticals, Burlingame, CA, USA).

\section{Antistress effects of YKS}

\section{Rat model of social isolation-induced stress}

Effects of YKS on psychological stress were investigated using a social isolation model. A total of 21 rats were randomly divided into groups of seven as follows: 1) Con, 2) individually housed stress (Stress), and 3) YKS (300 mg/kg)-treated 


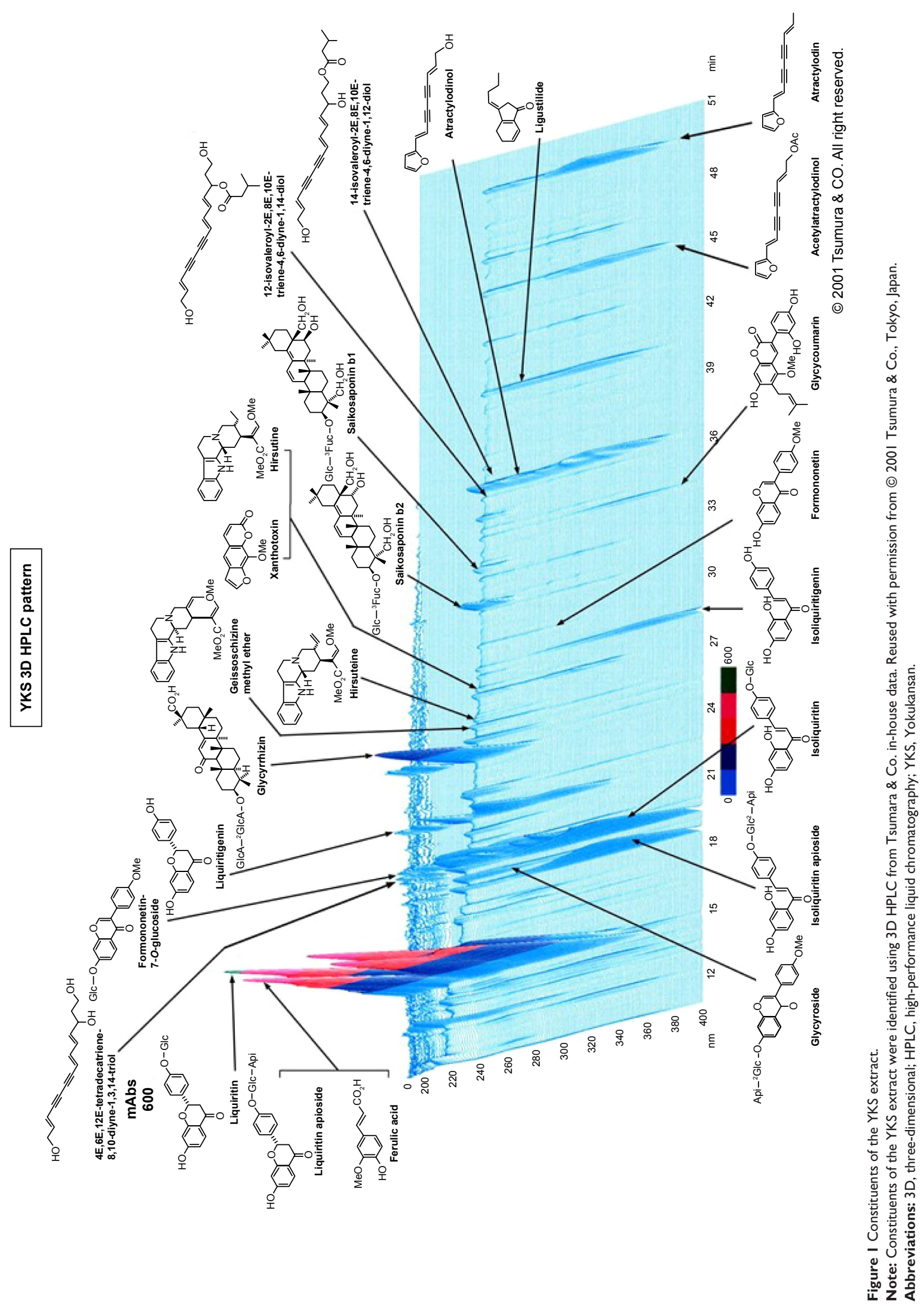


stress (Stress + YKS). Rats in the Stress and the Stress + YKS groups were individually housed for 8 days, and those in the Con group were housed in groups of two or three per cage. YKS (300 mg/kg) was orally administered to the Stress + YKS group daily for 7 days, and water was administered orally to the other groups.

\section{Food intake measurement}

Orexin is reported to control eating behavior, ${ }^{3}$ and the $24 \mathrm{~h}$ food intake was measured at 1:00 pm on days 2 and 7, according to a previous study. ${ }^{18}$ For the group-housed rats, the total food intake per cage was divided by the number of animals to calculate the food intake per animal for each cage, because food intake of individual rats was impossible to measure.

\section{Aggressive behavior test}

On day 7, aggressive behavior was analyzed according to a published method. ${ }^{12}$ Male rats (6-week-old, weighing 150-160 g) were housed in a group of three and used as intruders. An intruder rat was placed in a resident's home cage, and resident-intruder interactions were recorded using a video camera for $10 \mathrm{~min}$. Resident rats subjected to social isolation stress showed aggressive behavior characterized by wrestling, boxing, and biting. Tail rattling, lateral threat, and pursuit were excluded. The time spent exhibiting aggressive behavior was measured during a 10 min observation period. This test was performed from 1:00 to 3:00 pm.

\section{Plasma corticosterone levels}

Levels of plasma corticosterone were measured to evaluate psychological stress. ${ }^{16}$ On day 8 , rats were anesthetized using intraperitoneally administered pentobarbital sodium $(50 \mathrm{mg} / \mathrm{kg})$, and blood samples were taken from the inferior vena cava. All blood samplings were performed from 1:00 to 3:00 pm. Corticosterone levels were measured using an ELISA kit (ADI-900-097; Enzo Life Sciences, Farmingdale, NY, USA).

\section{Influences of social isolation stress on orexin secretion}

On day 8, levels of plasma orexins A and B (S-1148; Peninsula Laboratories International, San Carlos, CA, USA) were measured using ELISA kits. Next, orexin secretion in the lateral hypothalamus was determined using immunofluorescence analysis. After blood sampling, rats were exsanguinated by intracardial perfusion of phosphate-buffered saline (PBS) at $\mathrm{pH}$ 7.4. Rat brains were harvested after perfusion with $4 \%$ paraformaldehyde in $0.1 \mathrm{M}$ PBS. Tissue specimens were embedded in optimal cutting temperature compound (Sakura
Finetek Tissue-Tek, Tokyo, Japan), frozen, and then cut into $20 \mu \mathrm{m}$-thick sections using a cryostat (CM3050S; Leica Biosystems, Nussloch, Germany). Sections were incubated overnight at $4^{\circ} \mathrm{C}$ with a rabbit anti-orexin A antibody diluted 1:100 (PC362, Anti-Orexin A Rabbit Antibody; Merck, Darmstadt, Germany) and then incubated with Alexa Fluor 555conjugated donkey anti-rabbit IgG secondary antibody diluted 1:1,000 (Thermo Fisher Scientific, Waltham, MA, USA) for $2 \mathrm{~h}$ at room temperature. Slides were mounted in Vectashield Antifade Mounting Medium (H-1000; Vector Laboratories, Burlingame, CA, USA) and imaged using a confocal laser scanning fluorescence microscope (FV1000D; Olympus, Tokyo, Japan). The optical densities of immunoreactive staining were measured using an appropriate software program (FV10-AW; Olympus). All values were reported as an average of four micrographs per rat. Background immunofluorescence was measured in the absence of primary antibody.

\section{Involvement of orexin in stress responses}

To determine whether orexin induces stress responses, 15 rats were randomly divided into groups of five as follows: 1) Con, 2) Stress, and 3) orexin receptor antagonist-treated stress (Stress + DORA). Rats in the Stress and Stress + DORA groups were individually housed for 8 days, and those in the Con group were housed in groups of two or three per cage. Rats in the Stress + DORA group were administered DORA $(10 \mathrm{mg} / \mathrm{kg}) 1 \mathrm{~h}$ before the stress measurement on days 7 and 8 , and those in the other groups were administered only $50 \%$ dimethyl sulfoxide. On day 7, an aggressive behavior test was performed, ${ }^{12}$ and on day 8 , levels of plasma corticosterone were measured.

\section{Statistical analysis}

All experimental data are presented as mean \pm standard error of the mean (SEM). Statistical significance of differences in the data was evaluated using one-way analysis of variance (ANOVA) test. Comparisons between the three groups were performed using the post hoc Tukey test. All $P$-values $<0.05$ were considered statistically significant.

\section{Results}

\section{Influence of YKS on orexin secretion}

The administration of $300 \mathrm{mg} / \mathrm{kg}$ YKS for 7 days significantly decreased plasma orexin A level compared with that of the Con group $(254.06 \pm 75.54 \mathrm{pg} / \mathrm{mL}$ vs $386.29 \pm 55.58 \mathrm{pg} / \mathrm{mL}$, respectively; $P<0.05$ ), whereas the administration of $1,000 \mathrm{mg} / \mathrm{kg}$ YKS did not (356.80 $\pm 70.21 \mathrm{pg} / \mathrm{mL}$; Figure 2). Therefore, an effective dose of $300 \mathrm{mg} / \mathrm{kg}$ YKS was administered to rats in subsequent experiments. 


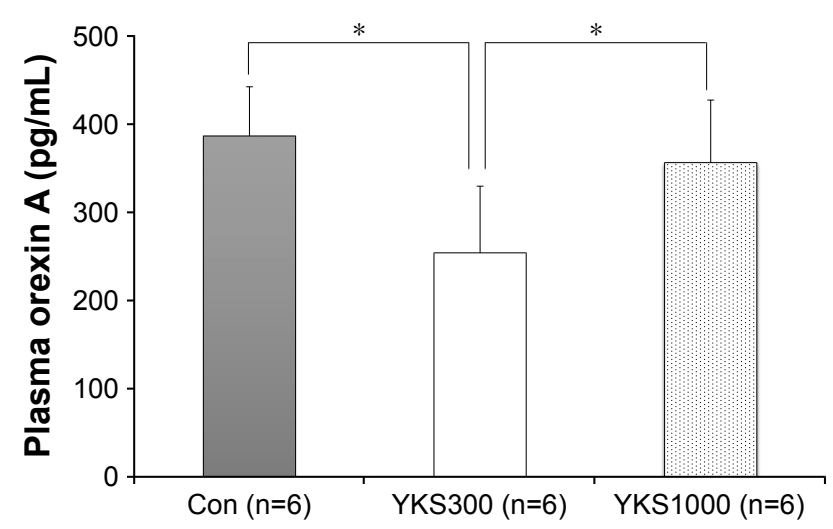

Figure 2 Plasma orexin A levels ( $\mathrm{pg} / \mathrm{mL}$ ) following the administration of YKS. Notes: Data are presented as mean \pm SEM. Statistical analysis: one-way ANOVA followed by Tukey's post hoc test, $* P<0.05$. Con, group-housed control. Abbreviations: ANOVA, analysis of variance; SEM, standard error of the mean; YKS300, Yokukansan (300 mg/kg); YKSI000, Yokukansan (I,000 mg/kg)

\section{Antistress effects of YKS}

Food intake measurement

On days 2 and 7, the $24 \mathrm{~h}$ food intake was measured. There rats were included in the Con group because seven rats were divided into three cages. On day 2 , no difference was observed among the three groups. On day 7 , the food intake in the Stress group $(22.76 \pm 2.01 \mathrm{~g})$ tended to increase with time, although this difference was not statistically significant compared with that in the Con group $(21.51 \pm 0.84 \mathrm{~g})$ and the Stress + YKS group (21.59 \pm 0.73 ; Figure 3A).

\section{Aggressive behavior test}

On day 7, we measured the total duration of aggressive behavior during a $10 \mathrm{~min}$ observation. Rats in the Stress group exhibited significantly longer aggressive behavior compared with that of rats in the Con group $(172.29 \pm 43.52 \mathrm{~s}$ vs $5.71 \pm 6.90 \mathrm{~s}$, respectively; $P<0.01$ ); however, YKS administration significantly inhibited aggressive behavior $(90.00 \pm 46.21 \mathrm{~s}, P<0.01$; Figure 3B).

\section{Plasma corticosterone level}

On day 8, plasma corticosterone levels were measured as a marker of stress. Plasma corticosterone level significantly increased in the Stress group compared with that in the Con group $(69.81 \pm 26.14 \mathrm{ng} / \mathrm{mL}$ vs $25.57 \pm 19.30 \mathrm{ng} / \mathrm{mL}$, respectively; $P<0.01)$; however, YKS administration significantly

A

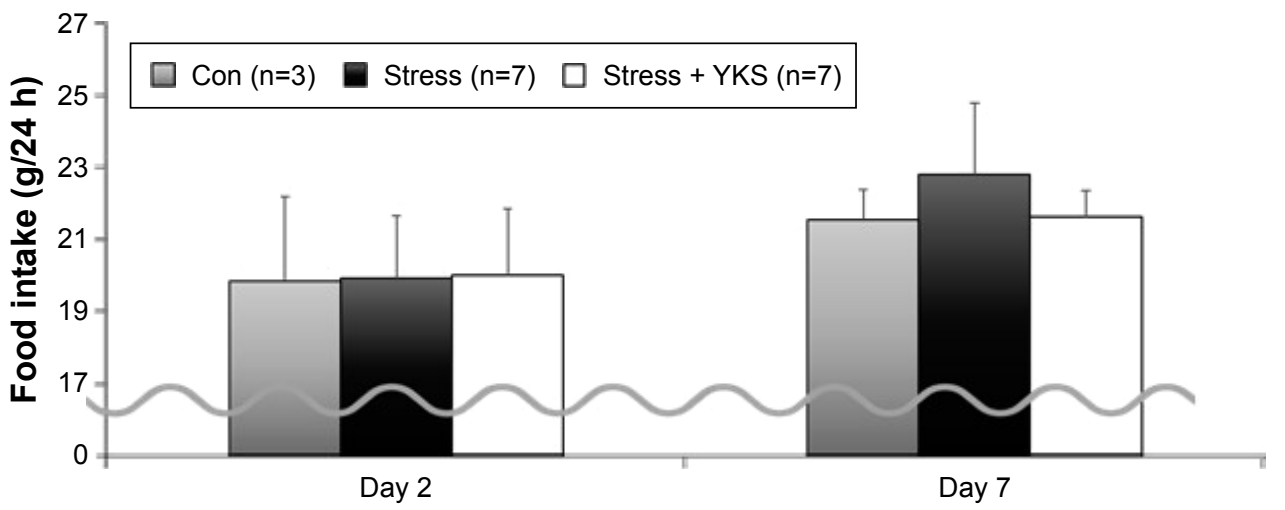

B

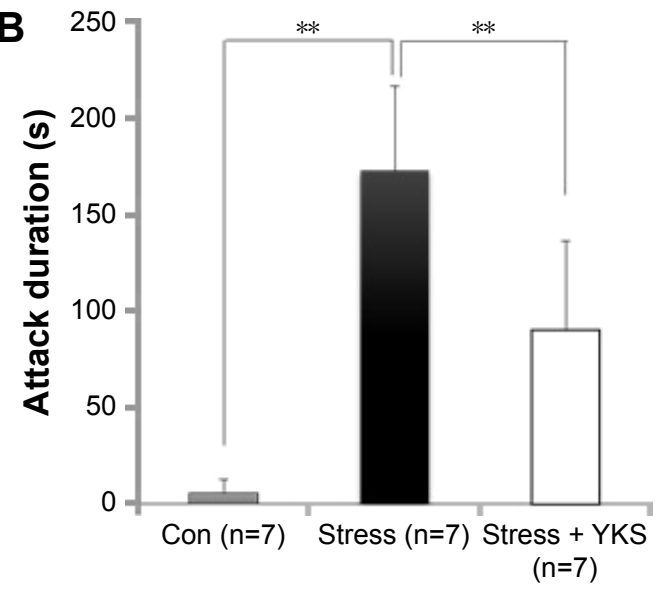

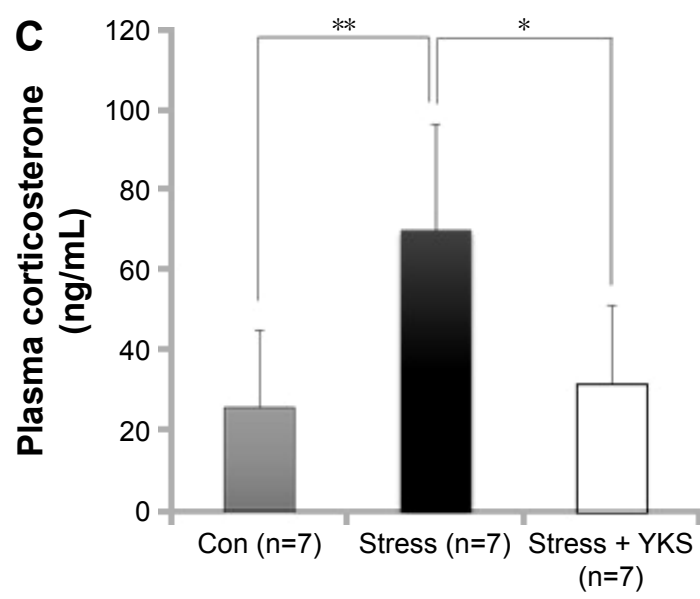

Figure 3 Effects of YKS on social isolation stress.

Notes: Food intake (g) (A), attack duration (s) in the resident-intruder test (B) and plasma corticosterone levels (ng/mL) $(\mathbf{C})$. Data are presented as mean \pm SEM. Statistical analysis: one-way ANOVA followed by Tukey's post hoc test, $* P<0.05$, $* * P<0.0$ I. Con, group-housed control; Stress, individually housed stress; Stress + YKS, individually housed YKS-treated stress.

Abbreviations: ANOVA, analysis of variance; SEM, standard error of the mean; YKS, Yokukansan. 
inhibited the elevation of corticosterone level in response to stress (31.49 $\pm 19.54 \mathrm{ng} / \mathrm{mL}, P<0.05$; Figure 3C).

\section{Influence of social isolation stress on orexin secretion}

On day 8, orexin A levels were significantly higher in the Stress group than in the Con group $(530.09 \pm 31.86 \mathrm{pg} / \mathrm{mL}$ vs $343.52 \pm 137.67 \mathrm{pg} / \mathrm{mL}$, respectively; $P<0.05$ ); however, YKS administration significantly inhibited the elevation in orexin A level (337.52 $\pm 122.33 \mathrm{pg} / \mathrm{mL}, P<0.05$; Figure 4A). Similarly, orexin B levels were significantly higher in the Stress group than in the Con group $(538.57 \pm 92.60 \mathrm{pg} / \mathrm{mL}$ vs $442.99 \pm 28.25 \mathrm{pg} / \mathrm{mL}$, respectively; $P<0.05$ ); however, YKS administration significantly inhibited the elevation in orexin B level in response to stress $(432.55 \pm 68.89 \mathrm{pg} / \mathrm{mL}$; $P<0.05$; Figure 4B). Orexin A and B secretion levels were similar.

Immunohistochemical analysis revealed increased number of orexin A $(+)$ cells in the lateral hypothalami of rats in the Stress group compared with the Con group (Figure 4C); however, this elevation in the number of cells was inhibited in the Stress + YKS group. The values were then expressed as optical densities (Figure 4D). Four images were recorded per rat, the immune reactivities were quantified, and all values were reported as an average of four micrographs per rat. The level was significantly increased in the Stress group (59.46 \pm 8.63 immunoreactive [IR] density) compared with that in the Con group $(37.99 \pm 10.22$ IR density; $P<0.01)$. This increase was significantly inhibited in the Stress + YKS group (47.48 \pm 5.46 IR density; $P<0.05$ ). Overall, these findings indicate that YKS attenuates the enhancement of orexin secretion caused by social isolation stress.

\section{Involvement of orexin in stress responses Aggressive behavior test}

DORA was administered to rats subjected to stress to investigate whether orexin was involved in stress responses. Rats in the Stress group exhibited aggressive behavior for significantly longer times compared with the Con group

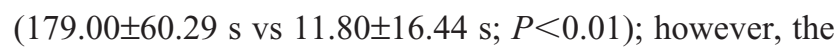
administration of DORA significantly inhibited aggressive

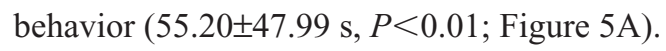

\section{Plasma corticosterone level}

Plasma corticosterone levels in the Stress group were significantly higher than those in the Con group $(73.11 \pm 35.67 \mathrm{ng} / \mathrm{mL}$ vs $25.63 \pm 5.73 \mathrm{ng} / \mathrm{mL}$, respectively; $P<0.05)$; however, YKS administration significantly inhibited the elevation of
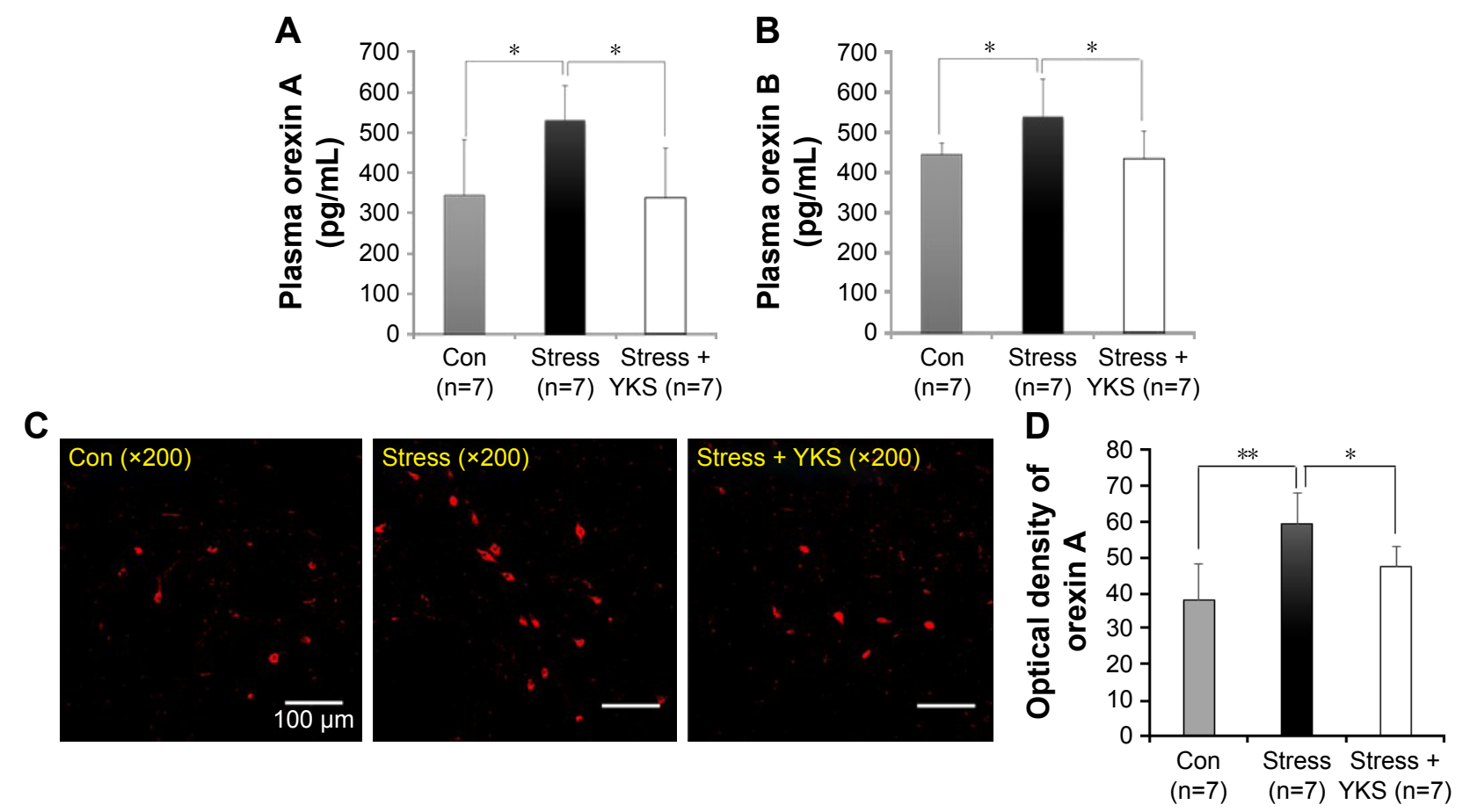

Figure 4 Effects of YKS on social isolation stress.

Notes: Plasma orexin A $(\mathbf{A})$ and B levels $(\mathrm{pg} / \mathrm{mL})(\mathbf{B})$. Orexin A $(+)$ cell (red) in the lateral hypothalamus; magnification, $\times 200 ;$ scale bar $=100 \mu \mathrm{m}(\mathbf{C})$. The fluorescence density quantification of orexin $A$ immune reactivity (D). Data are presented as mean \pm SEM. Statistical analysis: one-way ANOVA followed by Tukey's post hoc test, $* P<0.05$, $* * P<0.01$. Con, group-housed control; Stress, individually housed stress; Stress + YKS, individually housed YKS-treated stress.

Abbreviations: ANOVA, analysis of variance; SEM, standard error of the mean; YKS, Yokukansan. 

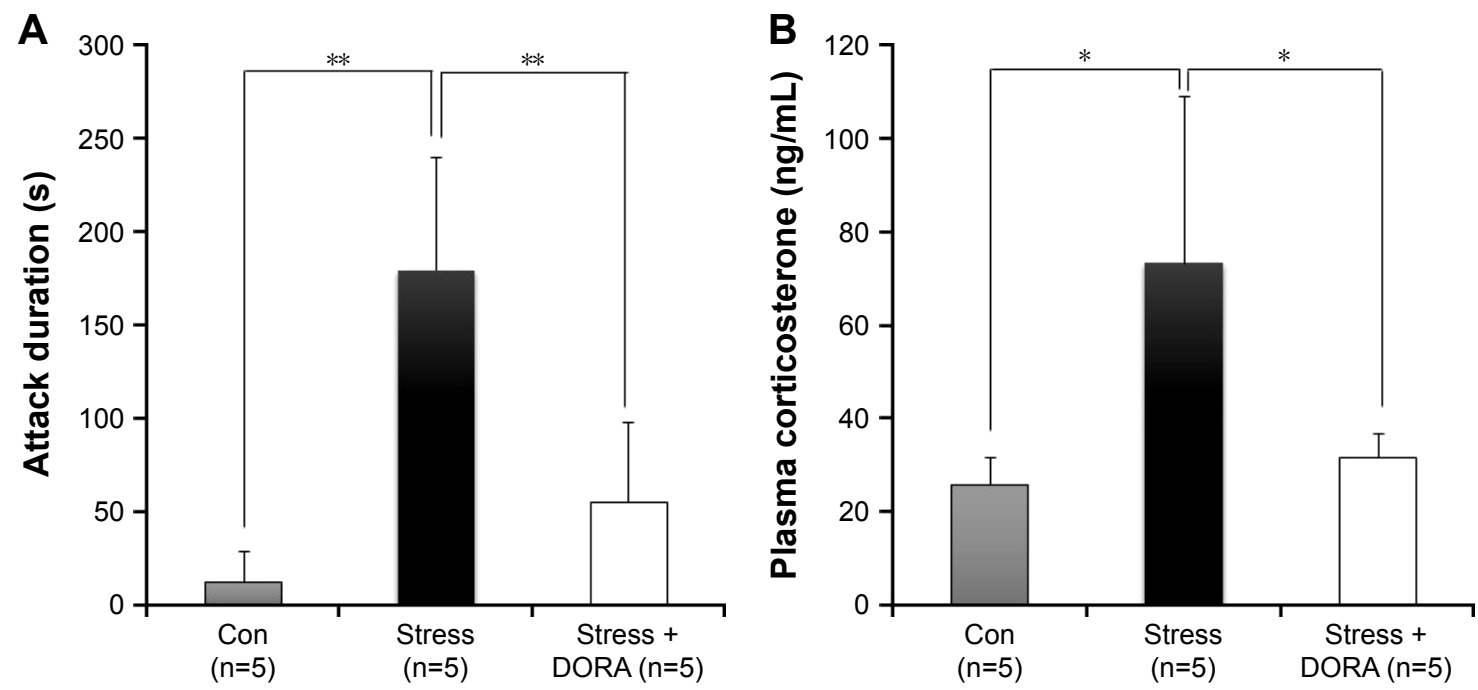

Figure 5 Effects of TCS I 102, a DORA, on social isolation stress.

Notes: Attack duration (s) in the resident-intruder test $(\mathbf{A})$ and plasma corticosterone levels $(\mathrm{ng} / \mathrm{mL})(\mathbf{B})$. Data are presented as mean \pm SEM. Statistical analysis: oneway ANOVA followed by Tukey's post hoc test, $* P<0.05$, $* * P<0.0$ I. Con, group-housed control; Stress, individually housed stress; Stress + DORA, dual orexin receptor antagonist-treated stress.

Abbreviations: ANOVA, analysis of variance; DORA, dual orexin receptor antagonist; SEM, standard error of the mean.

corticosterone levels in response to stress $(31.32 \pm 5.08 \mathrm{ng} / \mathrm{mL}$, $P<0.05$; Figure 5B).

\section{Discussion}

YKS exerts therapeutic effects on the behavioral and psychological symptoms of dementia (BPSD) of elderly people with dementia, ${ }^{26}$ and therefore it has received much attention. YKS has been reported to have antistress, ${ }^{14,15}$ anxiolytic, ${ }^{27}$ and antihallucinatory effects. ${ }^{28}$ Furthermore, it ameliorates sleep disorders ${ }^{24,25}$ and cognitive dysfunctions $;{ }^{29}$ acts as a neuroprotective agent, ${ }^{30}$ and induces neuronal differentiation. ${ }^{31}$ YKS is also an analgesic ${ }^{21,22,32}$ that prevents the development of morphine tolerance, ${ }^{33}$ and it acts as an antipruritic ${ }^{34}$ and inhibits allergic responses ${ }^{34}$ and dyskinesia. ${ }^{23}$

YKS is an agonist of the serotonin receptor 5hydroxytryptamine $(5-\mathrm{HT})_{1 \mathrm{~A}}{ }^{27}$ and inhibits the expression of $5-\mathrm{HT}_{2 \mathrm{~A}}$ receptors expressed by serotonergic and glutaminergic neurons in the central nervous system. ${ }^{28}$ Further, YKS inhibits the secretion of glutamate, ${ }^{35}$ enhances glutamate clearance from the extracellular fluid by astrocytes, ${ }^{36}$ and acts as an N-methyl-D-aspartate receptor antagonist. ${ }^{37}$ Moreover, YKS inhibits the activation of glial cells. ${ }^{32,33}$ However, to our knowledge, the effects of YKS on the interactions of orexin with components of the nervous system have not been previously reported. Therefore, in the current study, we investigated whether YKS has any influence on orexin secretion and whether the control of the interactions of orexin with the nervous system is involved in the antistress activity of YKS.
First, we administered YKS to rats that were not subjected to stress load for 1 week and then determined the effects of YKS on orexin secretion. The dose was determined according to the results of previous studies that administered YKS in the range of $50 \mathrm{mg} / \mathrm{kg}$ per day to $1,000 \mathrm{mg} / \mathrm{kg}$ per day, ${ }^{11-15,20-25}$ depending on the experimental model and animal species. Uchida et al, ${ }^{11}$ reported a stress load consistent with that of the current study and that $300 \mathrm{mg} / \mathrm{kg}$ of YKS was effective. In other experiments, YKS was administered at the upper limit of $1,000 \mathrm{mg} / \mathrm{kg} .{ }^{14,15,20,21}$ In this study, we orally administered these two doses once each day. Plasma orexin A concentration in the $300 \mathrm{mg} / \mathrm{kg}$ YKS-treated group was significantly lower than that of the Con group after 1 week; however, no difference between the two groups was observed for a YKS dose of $1,000 \mathrm{mg} / \mathrm{kg}$ (Figure 1). In general, therapeutic actions of drugs approved, for example, by the regulatory agencies of North American and European countries, are unidirectional and they are characterized by sigmoid dose-response curves. In contrast, because Kampo medicines comprise several types of crude medicinals, and as described earlier, its constituents have numerous and diverse pharmacological actions, agonists and antagonists coexist, and certain dose-response curves are not sigmoidal but peak in the middle of the concentration ranges tested. ${ }^{22,38}$

Next, we investigated the effects of YKS on stress response using a rat model of social isolation. In the current study, we investigated changes in food intake, aggressive behavior, and plasma corticosterone levels. Similar to that reported by a previous study, ${ }^{18}$ the food intake on day 7 in 
the Stress group tended to increase with time, although the difference was not statistically significant compared with that in the Con group. The time to engage in aggressive behavior in the presence of intruders was significantly extended by subjecting the rats to social isolation stress load for 1 week. Herein, we show that corticosterone secretion significantly increased in response to stress and that this stress effect was significantly inhibited by the administration of YKS (Figure 3A-C).

Subsequently, we investigated whether this stress response was involved in the regulation of orexin secretion. In experiments using model animals of stress, the activation of orexin neurons in the lateral hypothalamic region was induced by constraint, cold temperature, ${ }^{39}$ and forced swimming. ${ }^{40}$ Further, intracerebroventricular administration of orexin increases the levels of ACTH and corticosterone. ${ }^{41}$ In contrast, systemic administration of orexin acts directly on the adrenal cortex and increases plasma corticosterone and aldosterone levels. ${ }^{42}$ Moreover, orexin receptors are present in the adrenal medulla, ${ }^{43}$ and orexin promotes the secretion of epinephrine. ${ }^{44}$ These findings provide compelling evidence that orexin plays an important role in the stress response via the HPA and SAM axes.

Findings of the current study reveal that the significant increase in orexin level induced by social isolation-related stress load was significantly inhibited by the administration of YKS (Figure 4A and B). Xiao et $\mathrm{al}^{45}$ reported that the orexin A immunoreactivity in the lateral hypothalamus increased in rats with stress induced by electric foot shocks and noises. In the current study model as well, the excitability of orexin neurons in the hypothalamus was higher than that in the Con group, and this phenomenon was diminished by the administration of YKS (Figure 4C and D). Orexins A and B are synthesized from the same precursor prepro-orexin, and their secretion levels are similar. ${ }^{4}$ In the current study, we detected elevated secretion of orexins $\mathrm{A}$ and $\mathrm{B}$ in response to stress loading, which could be inhibited via the administration of YKS. Orexinergic neurons are mainly expressed in the lateral and perifornical areas of the posterior hypothalamus; however, they were found in other organs, such as gut, stomach, and pancreas. ${ }^{46}$ Therefore, more detailed studies are needed to clarify whether the inductions of these stress responses and the antistress effects of YKS are associated with orexin secretion in the hypothalamus alone.

Finally, we investigated whether orexin was involved in the induction of stress response. There are two orexin receptor isoforms $\mathrm{OX} 1 \mathrm{R}$ and $\mathrm{OX} 2 \mathrm{R} .{ }^{4}$ Administering a DORA to stress-loaded animals significantly inhibited aggression and increased plasma corticosterone levels, suggesting that orexin was involved in the induction of stress response. The differences, if any, in the actions of orexin A and B and OX1R and $\mathrm{OX} 2 \mathrm{R}$ are unknown, but we plan to conduct experiments in the future to determine whether they have separate roles in the stress response. Our current findings suggest that orexin is involved in the induction of stress response to social isolation-related stress load and that YKS may exhibit its antistress effect through the regulation of orexin secretion. Because only aggressive behavior and plasma corticosterone level were investigated as stress responses in this study, further studies are necessary to elucidate the associations between other stress responses and orexin and the efficacy of YKS on them.

YKS comprises seven crude medicinals (Table 1). According to Kampo medical theory, of these seven crude medicinals, Bupleuri radix, Cnidii rhizoma, and Uncariae cum Uncis ramulus are used to stabilize the mind. Orexin neurons are inhibited by serotonin (5-HT), noradrenalin, and gamma-aminobutyric acid, and the action of 5-HT is transduced through the $5-\mathrm{HT}_{1 \mathrm{~A}}$ receptor. ${ }^{4,47}$

YKS is an agonist of the $5-\mathrm{HT}_{1 \mathrm{~A}}$ receptor, and evidence indicates that geissoschizine methyl ether, an alkaloid synthesized by Uncariae cum Uncis ramulus, ${ }^{20,27}$ mediates this activity. Accordingly, the action of Uncariae cum Uncis ramulus via the $5-\mathrm{HT}_{1 \mathrm{~A}}$ receptor may represent one of the mechanisms through which YKS exerts its inhibitory effects on orexin neurons. Future studies are required to identify crude medicinals that regulate orexin secretion as well as their mechanisms.

Orexin neurons integrate information received from tissues, such as the limbic system, hypothalamus, and brainstem, and adjust behavior and autonomic functions. Therefore, certain disorders of these autonomic functions are caused by malfunction of the orexin system. To the best of our knowledge, the current study is the first to investigate the effects of YKS on the regulatory action of the orexin system, and our findings will likely form the basis of future experiments that will help elucidate indications for using YKS.

\section{Conclusion}

Our findings suggest that orexin is involved in the regulation of some stress responses and that YKS exerts its antistress effect via the regulation of orexin secretion.

\section{Acknowledgment}

The authors are grateful to Tsumura \& Co. for generously providing the YKS. 


\section{Disclosure}

The authors report no conflicts of interest in this work.

\section{References}

1. López M, Tena-Sempere M, Diéguez C. Cross-talk between orexins (hypocretins) and the neuroendocrine axes (hypothalamic-pituitary axes). Front Neuroendocrinol. 2010;31(2):113-127.

2. Kuwaki T. Orexin links emotional stress to autonomic functions. Auton Neurosci. 2011;161(1-2):20-27.

3. Messina G, Dalia C, Tafuri D, et al. Orexin-A controls sympathetic activity and eating behavior. Front Psychol. 2014;5:997.

4. Sakurai T. The neural circuit of orexin (hypocretin): maintaining sleep and wakefulness. Nat Rev Neurosci. 2007;8(3):171-181.

5. Watanabe S, Kuwaki T, Yanagisawa M, Fukuda Y, Shimoyama M. Persistent pain and stress activate pain-inhibitory orexin pathways. Neuroreport. 2005;16(1):5-8.

6. Yamamoto T, Nozaki-Taguchi N, Chiba T. Analgesic effect of intrathecally administered orexin-A in the rat formalin test and in the rat hot plate test. Br J Pharmacol. 2002;137(2):170-176.

7. Shirasaka T, Nakazato M, Matsukura S, Takasaki M, Kannan H. Sympathetic and cardiovascular actions of orexins in conscious rats. Am J Physiol. 1999;277(6 pt 2):R1780-R1785.

8. Young JK, Wu M, Manaye KF, et al. Orexin stimulates breathing via medullary and spinal pathways. J Appl Physiol. 2005;98(4):1387-1395.

9. Jászberényi M, Bujdosó E, Pataki I, Telegdy G. Effects of orexins on the hypothalamic-pituitary-adrenal system. J Neuroendocrinol. 2000; 12(12):1174-1178.

10. de Caires S, Steenkamp V. Use of Yokukansan (TJ-54) in the treatment of neurological disorders: a review. Phytother Res. 2010;24(9): 1265-1270.

11. Uchida N, Egashira N, Iwasaki K, et al. Yokukansan inhibits social isolation-induced aggression and methamphetamine-induced hyperlocomotion in rodents. Biol Pharm Bull. 2009;32(3):372-375.

12. Takeda A, Iwaki H, Ide K, Tamano H, Oku N. Therapeutic effect of Yokukansan on social isolation-induced aggressive behavior of zincdeficient and pair-fed mice. Brain Res Bull. 2012;87(6):551-555.

13. Tamano H, Kan F, Oku N, Takeda A. Ameliorative effect of Yokukansan on social isolation-induced aggressive behavior of zincdeficient young mice. Brain Res Bull. 2010;83(6):351-355.

14. Shimizu S, Tanaka T, Tohyama M, Miyata S. Yokukansan normalizes glucocorticoid receptor protein expression in oligodendrocytes of the corpus callosum by regulating microRNA-124a expression after stress exposure. Brain Res Bull. 2015;114:49-55.

15. Shimizu S, Tanaka T, Takeda T, Tohyama M, Miyata S. The Kampo medicine Yokukansan decreases microRNA-18 expression and recovers glucocorticoid receptors protein expression in the hypothalamus of stressed mice. Biomed Res Int. 2015;2015:797280.

16. Miyashita T, Yamaguchi T, Motoyama K, Unno K, Nakano Y, Shimoi K. Social stress increases biopyrrins, oxidative metabolites of bilirubin, in mouse urine. Biochem Biophys Res Commun. 2006;349(2):775-780.

17. Gentsch C, Lichtsteiner M, Frischknecht HR, Feer H, Siegfried B. Isolation-induced locomotor hyperactivity and hypoalgesia in rats are prevented by handling and reversed by resocialization. Physiol Behav. 1988;43(1):13-16.

18. Yamada C, Saegusa Y, Nahata M, Sadakane C, Hattori T, Takeda H. Influence of aging and gender differences on feeding behavior and ghrelin-related factors during social isolation in mice. PLoS One. 2015; 10(10):e0140094.

19. Ortiz GG, Zúñiga-González G, García JJ, Torres-Bugarín O, Zamora-Perez A, Bitzer-Quintero OK. 1-Methyl-4-phenyl-1,2,3,6tetrahydropyridine-induced lipid peroxidation and DNA damage in mouse bone marrow and blood. Environ Mol Mutagen. 2003;41(1):64-68.

20. Nishi A, Yamaguchi T, Sekiguchi K, et al. Geissoschizine methyl ether,

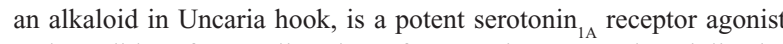
and candidate for amelioration of aggressiveness and sociality by Yokukansan. Neuroscience. 2012;207:124-136.
21. Suzuki Y, Mitsuhata H, Yuzurihara M, Kase Y. Antiallodynic effect of herbal medicine Yokukansan on peripheral neuropathy in rats with chronic constriction injury. Evid Based Complement Alternat Med. 2012;2012:953459.

22. Koshikawa N, Imai T, Takahashi I, Yamauchi M, Sawada S, Kansaku A. Effects of Hochu-ekki-to, Yoku-kan-san and Saiko-ka-ryukotsu-borei-to on behavioral despair and acetic acid-induced writhing in mice. Methods Find Exp Clin Pharmacol. 1998;20(1):47-51.

23. Sekiguchi K, Kanno H, Yamaguchi T, Ikarashi Y, Kase Y. Ameliorative effect of Yokukansan on vacuous chewing movement in haloperidolinduced rat tardive dyskinesia model and involvement of glutamatergic system. Brain Res Bull. 2012;89(5-6):151-158.

24. Ogawa Y, Fujii Y, Sugiyama R, Konishi T. The role of the seven crude drug components in the sleep-promoting effect of Yokukansan. J Ethnopharmacol. 2016;177:19-27.

25. Egashira N, Nogami A, Iwasaki K, et al. Yokukansan enhances pentobarbital-induced sleep in socially isolated mice: possible involvement of GABA(A)-benzodiazepine receptor complex. J Pharmacol Sci. 2011; 116(3):316-320.

26. Iwasaki K, Satoh-Nakagawa T, Maruyama M, et al. A randomized, observer-blind, controlled trial of the traditional Chinese medicine YiGan San for improvement of behavioral and psychological symptoms and activities of daily living in dementia patients. $J$ Clin Psychiatry. 2005;66(2):248-252.

27. Yamaguchi T, Tsujimatsu A, Kumamoto H, et al. Anxiolytic effects of Yokukansan, a traditional Japanese medicine, via serotonin 5-HT receptors on anxiety-related behaviors in rats experienced aversive stress. J Ethnopharmacol. 2012;143(2):533-539.

28. Egashira N, Iwasaki K, Ishibashi A, et al. Repeated administration of Yokukansan inhibits DOI-induced head-twitch response and decreases expression of 5-hydroxytryptamine (5-HT)2A receptors in the prefrontal cortex. Prog Neuropsychopharmacol Biol Psychiatry. 2008;32(6):1516-1520.

29. Tabuchi M, Yamaguchi T, Iizuka S, Imamura S, Ikarashi Y, Kase Y. Ameliorative effects of Yokukansan, a traditional Japanese medicine, on learning and non-cognitive disturbances in the Tg2576 mouse model of Alzheimer's disease. J Ethnopharmacol. 2009;122(1):157-162.

30. Kawakami Z, Kanno H, Ikarashi Y, Kase Y. Yokukansan, a kampo medicine, protects against glutamate cytotoxicity due to oxidative stress in PC12 cells. J Ethnopharmacol. 2011;134(1):74-81.

31. Furuya M, Miyaoka T, Tsumori T, et al. Yokukansan promotes hippocampal neurogenesis associated with the suppression of activated microglia in Gunn rat. J Neuroinflammation. 2013;10:145.

32. Suga $H$, Sunagawa $M$, Ikemoto $H$, et al. The analgesic and anti-stress effects of a Kampo medicine (Yokukansan) in rats with chronic constriction injury - a comparative study with kamishoyosan. J Integr Med Ther. 2015;2(1):5.

33. Takemoto M, Sunagawa M, Okada M, et al. Yokukansan, a Kampo medicine, prevents the development of morphine tolerance through the inhibition of spinal glial cell activation in rats. Integr Med Res. 2016; 5(1):41-47.

34. Funakushi N, Yamaguchi T, Jiang J, et al. Ameliorating effect of Yokukansan on the development of atopic dermatitis-like lesions and scratching behavior in socially isolated NC/Nga mice. Arch Dermatol Res. 2011;303(9):659-667.

35. Takeda A, Itoh H, Tamano H, Yuzurihara M, Oku N. Suppressive effect of Yokukansan on excessive release of glutamate and aspartate in the hippocampus of zinc-deficient rats. Nutr Neurosci. 2008;11(1): $41-46$.

36. Kawakami Z, Ikarashi Y, Kase Y. Glycyrrhizin and its metabolite 18 beta-glycyrrhetinic acid in glycyrrhiza, a constituent herb of Yokukansan, ameliorate thiamine deficiency-induced dysfunction of glutamate transport in cultured rat cortical astrocytes. Eur J Pharmacol. 2010;626(2-3):154-158.

37. Kawakami Z, Ikarashi Y, Kase Y. Isoliquiritigenin is a novel NMDA receptor antagonist in kampo medicine Yokukansan. Cell Mol Neurobiol. 2011;31(8):1203-1212. 
38. Manabe N, Camilleri M, Rao A, et al. Effect of daikenchuto (TU-100) on gastrointestinal and colonic transit in humans. Am JPhysiol Gastrointest Liver Physiol. 2010;298(6):G970-G975.

39. Ida T, Nakahara K, Murakami T, Hanada R, Nakazato M, Murakami N. Possible involvement of orexin in the stress reaction in rats. Biochem Biophys Res Commun. 2000;270(1):318-323.

40. Chang H, Saito T, Ohiwa N, et al. Inhibitory effects of an orexin-2 receptor antagonist on orexin A- and stress-induced ACTH responses in conscious rats. Neurosci Res. 2007;57(3):462-466.

41. Kuru M, Ueta Y, Serino R, et al. Centrally administered orexin/hypocretin activates HPA axis in rats. Neuroreport. 2000;11(9):1977-1980.

42. Malendowicz LK, Hochol A, Ziolkowska A, Nowak M, Gottardo L, Nussdorfer GG. Prolonged orexin administration stimulates steroidhormone secretion, acting directly on the rat adrenal gland. Int J Mol Med. 2001;7(4):401-404.
43. Blanco M, García-Caballero T, Fraga M, et al. Cellular localization of orexin receptors in human adrenal gland, adrenocortical adenomas and pheochromocytomas. Regul Pept. 2002;104(1-3):161-165.

44. Nemoto T, Toyoshima-Aoyama F, Ueda Y, et al. Involvement of the orexin system in adrenal sympathetic regulation. Pharmacology. 2013; 91(5-6):250-258.

45. Xiao F, Jiang M, Du D, et al. Orexin A regulates cardiovascular responses in stress-induced hypertensive rats. Neuropharmacology. 2013;67: $16-24$.

46. Kukkonen JP, Holmqvist T, Ammoun S, Akerman KE. Functions of the orexinergic/hypocretinergic system. Am J Physiol Cell Physiol. 2002; 283(6):C1567-C1591.

47. Muraki Y, Yamanaka A, Tsujino N, Kilduff TS, Goto K, Sakurai T. Serotonergic regulation of the orexin/hypocretin neurons through the 5-HT ${ }_{1 \mathrm{~A}}$ receptor. J Neurosci. 2004;24(32):7159-7166.

\section{Publish your work in this journal}

Neuropsychiatric Disease and Treatment is an international, peerreviewed journal of clinical therapeutics and pharmacology focusing on concise rapid reporting of clinical or pre-clinical studies on a range of neuropsychiatric and neurological disorders. This journal is indexed on PubMed Central, the 'PsycINFO' database and CAS, and is the official journal of The International Neuropsychiatric Association (INA). The manuscript management system is completely online and includes a very quick and fair peer-review system, which is all easy to use. Visit http://www.dovepress.com/testimonials.php to read real quotes from published authors.

Submit your manuscript here: http://www.dovepress.com/neuropsychiatric-disease-and-treatment-journal 